\title{
Nanoscale
}

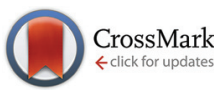

Cite this: Nanoscale, 2015, 7, 11328

\section{In situ investigation of two-step nucleation and growth of CdS nanoparticles from solution}

\author{
A. Schiener, ${ }^{\star a}$ A. Magerl, ${ }^{a}$ A. Krach, ${ }^{b}$ S. Seifert, ${ }^{c}$ H.-G. Steinrück, ${ }^{a}$ J. Zagorac, ${ }^{d}$ \\ D. Zahn ${ }^{d}$ and R. Weihrich ${ }^{b}$
}

\begin{abstract}
We report on a combined ultra-fast in situ SAXS and WAXS study along a free-jet providing insight into the evolution of the morphology and crystalline structure of CdS quantum dots in the very early stage of nucleation between $100 \mu \mathrm{s}$ and $2.5 \mathrm{~ms}$ with a time resolution down to $10 \mu \mathrm{s}$. Accessing this yet unexplored time regime provides direct evidence of a two-step mechanism via formation of prenucleation clusters followed by nanoparticle nucleation from coalescing precursors. Using ab initio calculations, the latter species is identified as $\mathrm{Cd}_{13} \mathrm{~S}_{4}(\mathrm{SH})_{18}$ clusters, the stability of which results from a compact surface and inner structure.
\end{abstract}

Received 12th March 2015

Accepted 16th May 2015

DOI: $10.1039 / c 5 n r 01602 a$

www.rsc.org/nanoscale

\section{Introduction}

Over the last few decades, both the scientific and the industrial interest in semiconducting nanoparticles (quantum dots) increased tremendously due to their unique optical, chemical and electrical properties. ${ }^{1-3}$ When the size of a semiconductor becomes smaller than the exciton radius of the bulk material, the electron hole pairs change their energy eigenstates to higher values. ${ }^{4,5}$ This quantum confinement occurs in quantum dots in all three dimensions of space and changes their properties significantly. ${ }^{6,7}$ For an efficient and controlled production of quantum dots various synthesis routes have been developed in order to tailor the particle characteristics by controlling their size and morphology. ${ }^{8}$ Besides the particle synthesis in gaseous environments ${ }^{9,10}$ precipitation of quantum dots from supersaturated solutions ${ }^{8}$ is a widely applied approach. Although ex situ and second-resolved in situ experiments can explore the nature of the precipitation at an advanced stage, the fundamental mechanisms driving the early stages of rapid particle formation are poorly understood as a consequence of the short time scale on which the unperturbed reaction takes place. ${ }^{11}$ In previous in situ investigations with a time resolution of seconds to several minutes the reac-

\footnotetext{
${ }^{a}$ Crystallography and Structural Physics, Friedrich-Alexander-Universität ErlangenNürnberg, Department of Physics, Staudtstraße 3, 91058 Erlangen, Germany. E-mail: andreas.schiener@fau.de

${ }^{b}$ Institute of Inorganic Chemistry, Universität Regensburg, Universitätsstraße 31, 93040 Regensburg, Germany

${ }^{c}$ Advanced Photon Source, Argonne National Laboratory, Chemistry Division, 9700 S. Cass Avenue, Argonne, IL 60439, US

${ }^{d}$ Theoretical Chemistry and Computer-Chemistry-Center (CCC), Friedrich-AlexanderUniversität Erlangen-Nürnberg, Nägelsbachstraße 25, 91052 Erlangen, Germany
}

tions were slowed down with complexing ligands, which additionally stabilize the quantum dots once they have reached a certain size. ${ }^{12,13}$ It can be suspected that the addition of stabilizers modifies the intrinsic mechanism of the nucleation and growth process significantly.

Cadmium sulfide (CdS) with a bulk band gap of $2.53 \mathrm{eV}$ is a representative candidate for a II-VI semiconductor. ${ }^{8}$ To access the timescale at which the natural CdS precipitation reaction in wet media takes place, we have pioneered diffraction experiments in a free-jet setup. ${ }^{14}$ Setups using laminar mixing to investigate calcium carbonate crystallization down to $100 \mu \mathrm{s}^{15,16}$ have been published earlier. However, as our interest is in highly diluted systems, only turbulent mixing provides a sufficiently large interface between the reactants to obtain a significant scattering signal at early stages of particle formation. ${ }^{17}$ Further improvements of the setup, e.g. the suppression of spraying by proper shaping of the nozzle of the micro mixer, were needed to investigate simultaneously the morphology and the crystalline structure via combined small angle X-ray scattering (SAXS) and wide angle X-ray scattering (WAXS). Such a study presents both atomistic insight into the very early stages of crystallization and a description of the particle morphology of the formation of CdS quantum dots already from $100 \mu$ s onwards. As subsequently shown, structure models of the prenucleation clusters and reasons for their relative stability can be obtained from the combination of the obtained experimental results and ab initio calculations.

\section{Experimental}

We have developed a novel setup to access reaction times for particle formation in liquid media as short as $100 \mu$ s (Fig. 1). 


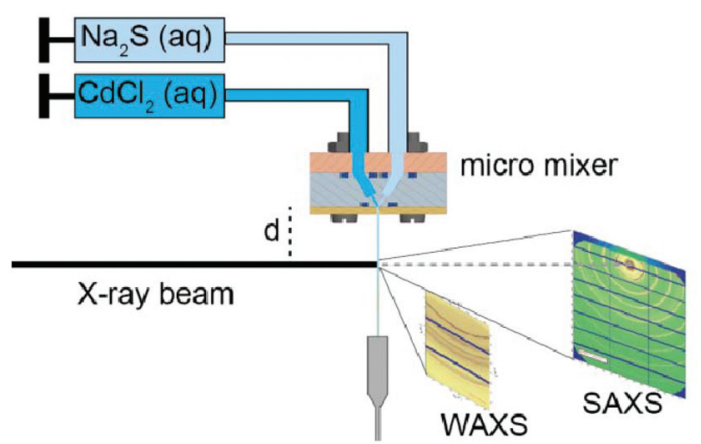

Fig. 1 Layout of the free-jet setup. Aqueous solutions of $\mathrm{Na}_{2} \mathrm{~S}$ and $\mathrm{CdCl}_{2}$ are brought together in a Y-shaped micro mixer and ejected as a continuous free-jet. A monochromatic X-ray beam (14 keV) is targeted at different positions along this jet and diffraction data in the SAXS and WAXS regime are acquired.

Two computer controlled high pressure stainless steel syringe pumps (Chemyx; Nexus 6000) are connected with high pressure tubes to the input channels of a micro Y-mixer with a short exit nozzle, which produces a free-jet of the mixed reactants cadmium chloride $\left(\mathrm{CdCl}_{2}\right)$ and sodium sulfide $\left(\mathrm{Na}_{2} \mathrm{~S}\right)$ in aqueous solution. Typically, the jet diameter is chosen between 200 and $500 \mu \mathrm{m}$. The formation of CdS quantum dots takes place along the continuous free-jet according to the chemical reaction

$$
\mathrm{CdCl}_{2}(\mathrm{aq})+\mathrm{Na}_{2} \mathrm{~S}(\mathrm{aq}) \rightarrow 2 \mathrm{NaCl}(\mathrm{aq})+\mathrm{CdS} \downarrow .
$$

By positioning a monochromatic X-ray beam (Advanced Photon Source, APS 12-ID-B station, photon energy $14 \mathrm{keV}$ ) at different positions along the free-jet, the simultaneous acquisition of small angle X-ray scattering (SAXS) and wide angle X-ray scattering (WAXS) data was achieved for different reaction times, $t_{\text {react }}$ which is calculated according to eqn.,

$$
t_{\text {react }}=\frac{d}{v}
$$

where $d$ and $v$ are the distance to the mixing point and the flow velocity, respectively. The distance from the beginning of the mixing of the reactants to the exit of the nozzle of the mixer is $1.3 \mathrm{~mm}$. The flow profile of the free-jet is assumed to have a rectangular profile, as appropriate for the conditions of turbulent flow. ${ }^{18}$

The time resolution depends on the flow velocity, and the uncertainty for the reaction time is dominated by the vertical size of the X-ray beam $(100 \mu \mathrm{m})$ and the radius of the bores at the mixing point $(200 \mu \mathrm{m})$ according to $\Delta t=\frac{300 \mu m}{v}$. This results in an exposure time independent uncertainty of the reaction time of about $15 \mu$ s for the data shown in Fig. 2 which were taken with a flow velocity of $21.2 \mathrm{~m} \mathrm{~s}^{-1}$. To exclude systematical errors in the data reduction the water background for every set of parameters was measured separately. In addition, a horizontal scan with 5 data points spaced by $100 \mu \mathrm{m}$

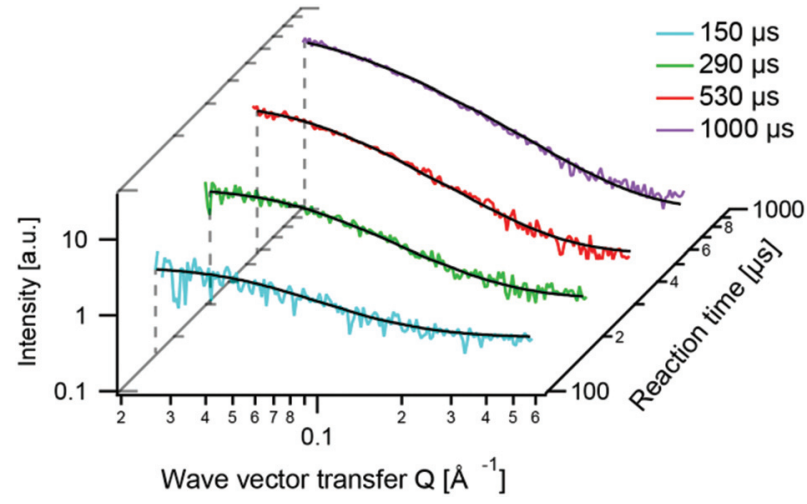

Fig. 2 Representative SAXS profiles acquired along the free-jet for reaction times of $150,290,530$, and $1000 \mu \mathrm{s}$ (flow velocity $21.2 \mathrm{~m} \mathrm{~s}^{-1}$, $\mathrm{Na}_{2} \mathrm{~S}$ and $\mathrm{CdCl}_{2}$ reactant concentrations $5 \mathrm{mM}$, exposure times $0.4 \mathrm{~s}$ ). The black lines show fits of the SAXS signal with a lognormal distributed spherical model for the data at 150, 290, and $530 \mu$ s and a bimodal lognormal distributed spherical model for the data at $1000 \mu \mathrm{s}$, as discussed in the text.

was taken at each vertical position to ensure that the X-ray beam targets the free-jet at the center.

The acquired 2D detector images (Dectris Pilatus 2M detector in the SAXS regime and Dectris Pilatus 300k detector in the WAXS regime) were reduced to radially integrated diffraction patterns using the Igor (Wavemetrics) extension Nika, ${ }^{19}$ and various model functions were fitted to these patterns using the Igor extension Irena. ${ }^{20}$

A systematic series of candidate $\mathrm{Cd}_{x} \mathrm{~S}_{y}(\mathrm{SH})_{z}$ clusters was characterized from quantum calculations. For this purpose, initial models were cut from the CdS single crystal structure based on a distance threshold $d$ from (1) a central 6-ring motif and (2) a $\mathrm{Cd}_{4} \mathrm{~S}$ tetrahedron. By variation of $d$ up to a few nanometers an increasing number of coordination spheres of the central motifs is included in the initial cluster model. To maintain charge neutrality, the outermost sulfur atoms were protonated.

The model clusters were then subjected to structural relaxation based on quantum calculations in a vacuum. For this, the Gaussian03 program package and the HF-DGDZVP basis set was used (M. J. Frisch, et al., Gaussian 03, Gaussian, Inc., Wallingford CT, 2004). The cluster geometry was optimized in two steps, first considering relaxation of the outer ions only and then performing energy minimization of the whole cluster in a vacuum.

In a next step the stability of the postulated clusters with respect to agglomeration was investigated from calculations with periodic boundary conditions. Two clusters were placed in a cell of $15 \times 15 \times 25 \AA^{3}$ size and the repulsion between them was calculated by moving them stepwise closer together. The cluster distance was adjusted by fixing the positions of the center cadmium atoms while the atomic positions of all other atoms were allowed to relax during the performance of geometry optimization. The quantum mechanical calculations were carried out within the framework of Density Functional Theory 
(DFT) with exchange-correlation functionals in the generalized gradient approximation (GGA) according to Perdew-BurkeErnzerhof (PBE). ${ }^{21,22}$ All calculations were executed with the Vienna Ab initio Simulation Package (VASP). ${ }^{23-26}$ Atomic site parameters were allowed to fully relax within the conjugant gradient algorithm. The interactions between ions and electrons were described by the projector-augmented-wave method $(\mathrm{PAW})^{27,28}$ with a cutoff energy of $500 \mathrm{eV}$. A structure optimization was considered to be converged with a difference in total energy of less than $10^{-6} \mathrm{eV}$ and a maximum HellmannFeynman force of $10^{-4} \mathrm{eV}^{-1}$.

\section{Results and discussion}

Fig. 2 shows a sequence of exemplary SAXS patterns acquired along the free-jet with a flow velocity of $21.2 \mathrm{~m} \mathrm{~s}^{-1}$ and a jet diameter of $400 \mu \mathrm{m}$. The distances to the mixing point are $3.3 \mathrm{~mm}, 6.3 \mathrm{~mm}, 11.3 \mathrm{~mm}$, and $21.3 \mathrm{~mm}$ giving reaction times of $150 \mu \mathrm{s}, 290 \mu \mathrm{s}, 530 \mu \mathrm{s}$, and $1000 \mu \mathrm{s}$, respectively. The exposure time was $0.4 \mathrm{~s}$ each. The spectra of the first three data points at $150 \mu \mathrm{s}, 290 \mu \mathrm{s}$, and $530 \mu \mathrm{s}$ can be reproduced with a lognormal distributed spherical model. In contrast, the pattern at $1000 \mu$ s clearly shows the appearance of a second length scale. This feature can be accounted for with the same level of goodness of fit by invoking either anisotropic particles (e.g. cylinder, disks and ellipsoidal particles), or by a bimodal lognormal distributed spherical size distribution. However, the bimodal spherical size distribution is the only model, which describes both the SAXS and the WAXS data, as presented in the next section.

A WAXS data set taken simultaneously with the SAXS data for a reaction time of $2480 \mu$ s (Fig. 3a) shows the presence of a broad maximum at about $1.7 \AA^{-1}$. The broad maximum represents the structure factor of an $\sim 1 \mathrm{~nm}$ sized structurally relaxed precursor cluster. Its diffraction pattern can be readily calculated using e.g. DISCUS, ${ }^{29}$ which is based on the Debye formalism and considers all pair correlations within the particle. We note that no narrow Bragg-like peak is found in the WAXS regime showing that all structures including the growing population are non-crystalline. ${ }^{30}$

We modeled the energy landscape of candidate precursor clusters by cutting fragments from the single crystal and neutralizing charge by removing/protonating $\mathrm{S}^{2-}$ ions at the surface. The candidate clusters were subjected to structural relaxation from quantum calculations and then benchmarked with respect to the experimental diffraction pattern. This procedure is illustrated for the best fit in Fig. 4. The resulting cluster may be interpreted as a $\mathrm{Cd}_{13} \mathrm{~S}_{4}(\mathrm{SH})_{18}$ complex and nicely reproduces the experimentally observed WAXS peak. Apart from the matching diffraction patterns, it should also be pointed out that the derived $\mathrm{Cd}_{13} \mathrm{~S}_{4}(\mathrm{SH})_{18}$ cluster reflects a practically spherical shape which suggests stabilization from favorable surface tension. Moreover, no under-coordination of $\mathrm{Cd}^{2+}$ or $\mathrm{S}^{2-}$ ions is observed which implies relatively low cluster reactivity. On the basis of both mechanical and chemi- a)

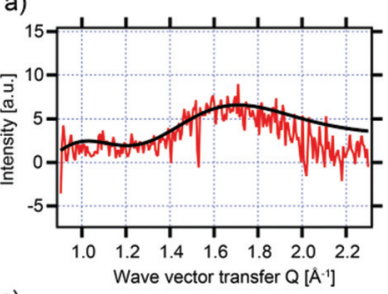

c)

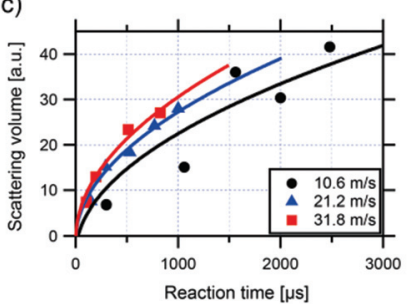

b)

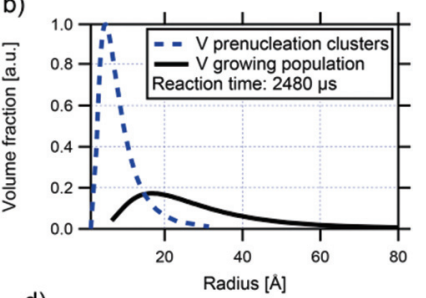

d)

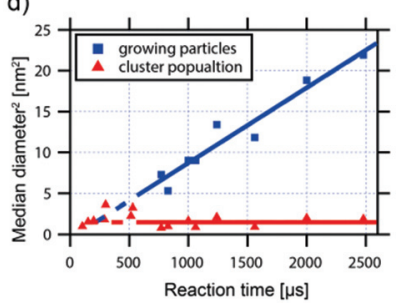

Fig. 3 (a) WAXS intensity for $2480 \mu$ s showing as a salient feature of a broad structure factor maximum at $Q \approx 1.7 \AA^{-1}$ (red). This is well produced in position and width by the calculated scattering function (black) for the relaxed precursor particle in Fig. 4b. (b) Fitted volume fractions of the SAXS data after a reaction time of $2480 \mu \mathrm{s}$. The dashed blue and the black lines show the contributions from the precursor clusters and from the growing particle population, respectively. (c) Total scattering volume for the SAXS data for three jet velocities $\left(10.6 \mathrm{~m} \mathrm{~s}^{-1}, 21.2 \mathrm{~m} \mathrm{~s}^{-1}\right.$, and $31.8 \mathrm{~m} \mathrm{~s}^{-1}$ ) as a function of reaction time. (d) Evolution of the squared median diameter of CdS particles with time (reactant concentration $5 \mathrm{mM})$, realized with 3 jet velocities $\left(10.6 \mathrm{~m} \mathrm{~s}^{-1}, 21.2 \mathrm{~m} \mathrm{~s}^{-1}\right.$, and $31.8 \mathrm{~m} \mathrm{~s}^{-1}$ ) and by various positions along the free-jet. The size of the growing particle population (blue squares) shows a square root dependence (solid blue line), characteristic for a diffusion driven growth. At all times exceeding $800 \mu$ s the presence of clusters with a size of about $1 \mathrm{~nm}$ is observed (solid red line). The dashed red and blue lines are extrapolations of the fits to the data points exceeding $800 \mu \mathrm{s}$.
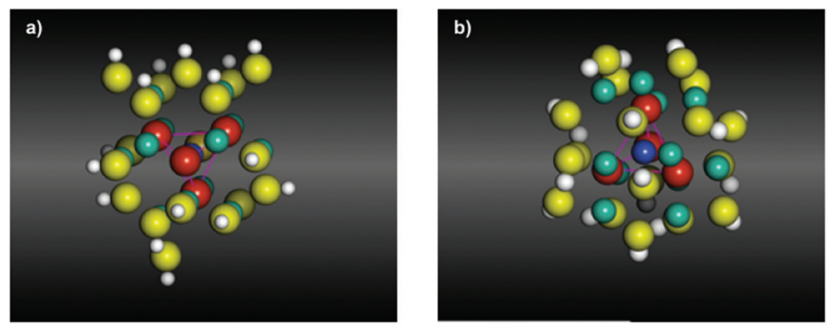

Fig. 4 (a) $\mathrm{Cd}_{13} \mathrm{~S}_{4}(\mathrm{SH})_{18}$ cluster derived from the CdS crystal structure using a distance threshold with respect to a central $\mathrm{CdS}_{4}$ motif (highlighted in blue and red, respectively). The outermost $\mathrm{S}^{2-}$ ions (yellow) were protonated to provide charge-neutral clusters. (b) Same cluster after energy minimization. While the coordination tetrahedra of the $\mathrm{Cd}^{2+}$ and $\mathrm{S}^{2-}$ ions are deformed, the $\mathrm{SH}^{-}$ions are drastically rearranged, resulting in a roughly spherical cluster. This not only ensures favorable surface tension, but also implies a 'passivation' of cluster reactivity by avoiding under-coordinated $\mathrm{S}^{2-}$ or $\mathrm{Cd}^{2+}$ ions.

cal arguments we thus suggest the $\mathrm{Cd}_{13} \mathrm{~S}_{4}(\mathrm{SH})_{18}$ cluster as a very reasonable proxy of the $1 \mathrm{~nm}$ sized clusters observed in the experiments.

In consequence, the SAXS data at $800 \mu$ s and later are to be described with a bimodal spherical model. Fig. 3b shows the 
volume fractions of the individual contributions to the SAXS signal of the small particle population dominant at high $Q$ values (blue) and of the big particle population dominant at small $Q$ values (black). While the small particle population shows constant size for all times, the size of the second particle population is growing with time (Fig. 3d).

The total scattering volume for both populations depicted in Fig. 3c shows an increase of the amount of particles formed with time. The strong turbulent mixing in the present setup leads to a large interface between the reactant solutions due to Kolmogorov eddies ${ }^{31}$ needed to create a sufficient amount of scattering volume for a reasonable SAXS signal at the short time scales considered here. We note that the volume increase with time becomes more pronounced with higher flow velocities, i.e. with stronger turbulent mixing. Still, the reaction on the short timescales considered here is dominated by diffusive processes across interfaces and not by mixing.

Based on these in situ SAXS/WAXS experiments we propose the following formation mechanism of CdS quantum dots at the early stages of crystallization illustrated in Fig. 5. $100 \mu \mathrm{s}$ after strong turbulent mixing the first stable clusters with a median diameter of $1 \mathrm{~nm}$ have already formed. This first nucleation process is driven by diffusion of the cadmium and sulfur ions and further nucleation continuously takes place in the regions of the interfaces of turbulent mixing. From these primary clusters larger particles develop. The median value of the diameter of these particles shows a square root dependence with time in support of a diffusion driven growth. The diffusion coefficient $D$ is calculated to be $1.5 \times 10^{-7} \mathrm{~cm}^{2} \mathrm{~s}^{-1}$ using the corresponding equations for diffusion limited growth after Ratke and Voorhees ${ }^{33}$ and the slope of the squared SAXS diameter of the growing particle population in

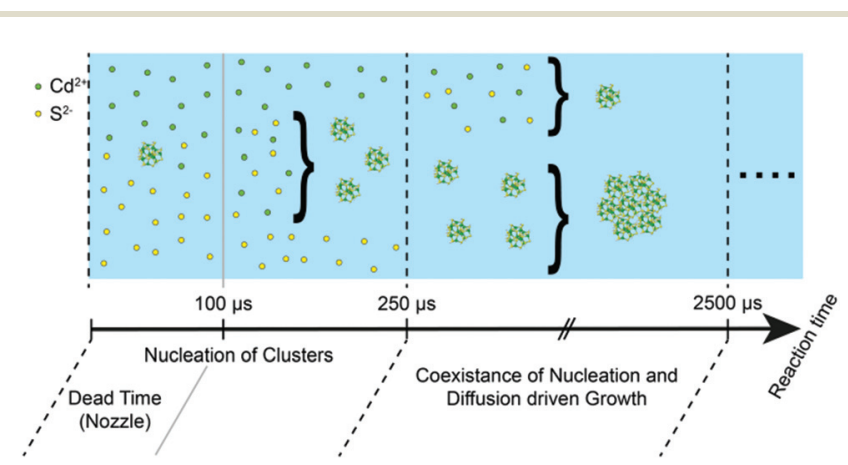

Fig. 5 Schematics of the formation mechanism of CdS quantum dots At $100 \mu \mathrm{s}$ (dead time of the setup) a small amount of clusters is already present. Up to $250 \mu$ s the median diameter of the early clusters remains constant indicating a high stability of the size of these clusters, while their concentration increases. A similar precursor stability has already been observed for $\mathrm{CaCO}_{3}$ prenucleation clusters by Gebauer et al. ${ }^{32}$ Prenucleation clusters thereby are introduced as ion complexes, forming before classical nucleation and growth sets in. Between $250 \mu \mathrm{s}$ and $2.5 \mathrm{~ms}$ a diffusion driven particle growth by clusters or particle attachment is observed. From about $800 \mu$ s onwards the particle growth becomes observable and a bimodal particle model is necessary to fit the SAXS data (blue line in Fig. 3d).
Fig. 3d. The value of the experimental diffusion constant $D$ is about two orders of magnitude smaller than typical ionic diffusion coefficients in aqueous solutions $\left(D\left(\mathrm{SH}^{-}\right)=1.73 \times\right.$ $10^{-5} \mathrm{~cm}^{2} \mathrm{~s}^{-1}$ and $\left.D\left(\mathrm{Cd}^{2+}\right)=0.719 \times 10^{-5} \mathrm{~cm}^{2} \mathrm{~s}^{-1}\right) \cdot{ }^{34}$ From this we argue that the growth proceeds through an attachment of clusters and not by attachment of atomic species. However, the understanding as to what happens on the atomistic scale during the attachment of small clusters is rather limited so far. ${ }^{35}$ After $2.5 \mathrm{~ms}$ the median of the particle diameter of the growing population has reached a value of about $5 \mathrm{~nm}$. Further growth and a transition into a long range crystalline state beyond that time scale is expected and is accessible by investigations with a standard stopped flow device. ${ }^{36}$ The presence of the $1 \mathrm{~nm}$ clusters at all times, in addition to the evaluated growth kinetics provides, to our knowledge, a first experimental indication for the existence of prenucleation clusters in rapid quantum dot formation.

In order to confirm the experimentally observed stability of the prenucleation clusters with respect to instantaneous agglomeration, this process was modeled with DFT calculations. One could estimate that the approach of two clusters initiates agglomeration and particle growth. However, the present calculations provide a different picture. We studied the change of the potential energy when two prenucleation clusters of the predicted composition $\mathrm{Cd}_{13} \mathrm{~S}_{4}(\mathrm{SH})_{18}$ (Fig. 4a) are moved closer stepwise. For approaching particles, repulsion is predicted from increasing potential energy and therefore the stability of single prenucleation clusters is confirmed. This is presented in Fig. 6 from a profile of the energy as a function of the distance of the centers of two clusters. An explanation for the increasing energy is given by the deformation of the coordination polyhedra which are closest to the neighboring cluster. This is illustrated by the red, blue, green and brown colored bonds in Fig. 6 .

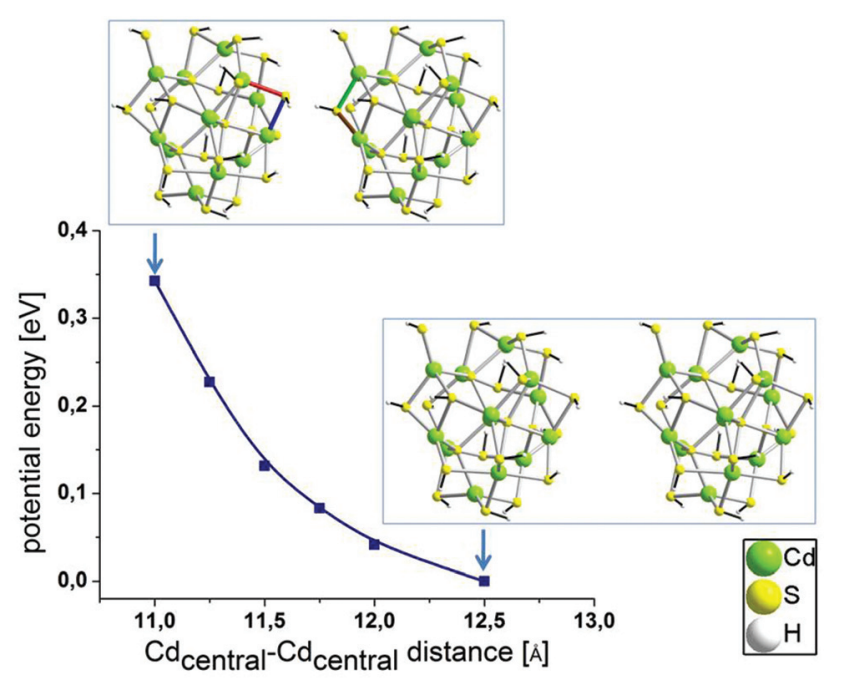

Fig. 6 Calculated structures and potential energy for two approaching clusters. 


\section{Conclusions}

This combined SAXS and WAXS study provides for the first time microsecond resolved direct experimental insights into the natural precipitation mechanism of CdS as a model system for a fast precipitation reaction. Experimental SAXS and WAXS data together with quantum calculations and DFT modelling support a bimodal size distribution on the so far unexplored timescale between $100 \mu \mathrm{s}$ and $2.5 \mathrm{~ms}$. The size of the small population remains constant during that timescale, while the second particle population is growing with time. The square root like growth behavior indicates a cluster diffusion driven particle growth mode and is a direct experimental proof of a 2-step nucleation process for the very early stages of CdS formation and growth. On the basis of quantum calculations we suggest $\mathrm{Cd}_{13} \mathrm{~S}_{4}(\mathrm{SH})_{18}$ as a structural model of the prenucleation cluster which (1) best fits the experimental diffraction data and (2) exhibits the compact shape and inner structure along with a relatively inert surface comprising of $\mathrm{SH}^{-}$ions.

\section{Acknowledgements}

We gratefully acknowledge funding by the German Research Foundation (DFG) through Priority Program SPP1415 and support by the Graduate School GRK 1896.

This research used resources of the Advanced Photon Source, a U.S. Department of Energy (DOE) Office of Science User Facility operated for the DOE Office of Science by Argonne National Laboratory under contract no. DE-AC02$06 \mathrm{CH} 11357$.

We furthermore gratefully acknowledge support by the APS 12 ID beamline staff.

\section{Notes and references}

$1 \mathrm{X}$. Michalet, et al., Quantum dots for live cells, in vivo imaging, and diagnostics, Science, 2005, 307, 538-544.

2 A. P. Alivisatos, Semiconductor Clusters, Nanocrystals, and Quantum Dots, Sci., 1996, 271, 933-937.

3 Y. Jun, J. Choi and J. Cheon, Shape control of semiconductor and metal oxide nanocrystals through nonhydrolytic colloidal routes, Angew. Chem., Int. Ed., 2006, 45, 34143439.

4 L. E. Brus, Electron-electron and electron-hole interactions in small semiconductor crystallites: The size dependence of the lowest excited electronic state, J. Chem. Phys., 1984, 80, 4403.

5 P. Lippens and M. Lannoo, Calculation of the band gap for small CdS and ZnS crystallites, Phys. Rev. B: Condens. Matter, 1989, 39, 10935-10942.

6 M. Nirmal, et al., Fluorescence intermittency in single cadmium selenide nanocrystals, Nature, 1996, 383, 802-804.
7 L. Brus, Electronic wave functions in semiconductor clusters: experiment and theory, J. Phys. Chem., 1986, 90, 2555-2560.

8 T. Trindade, P. O’Brien and N. L. Pickett, Nanocrystalline Semiconductors: Synthesis, Properties, and Perspectives, Chem. Mater., 2001, 13, 3843-3858.

9 G. Beaucage, et al., Probing the dynamics of nanoparticle growth in a flame using synchrotron radiation, Nat. Mater., 2004, 3, 370-374.

10 L. Mädler, W. J. Stark and S. E. Pratsinis, Rapid synthesis of stable ZnO quantum dots, J. Appl. Phys., 2002, 92, 6537.

$11 \mathrm{~J}$. Polte, et al., Nucleation and growth of gold nanoparticles studied via in situ small angle X-ray scattering at millisecond time resolution, ACS Nano, 2010, 4, 1076-1082.

12 F. Meneau, et al., In situ study of the formation of CdS nanoparticles by small-angle X-ray scattering, J. Appl. Crystallogr., 2003, 36, 718-721.

13 L. Qi, H. Cölfen and M. Antonietti, Synthesis and Characterization of CdS Nanoparticles Stabilized by DoubleHydrophilic Block Copolymers, Nano Lett., 2001, 1, 61-65.

14 A. Schiener, T. Wlochowitz, S. Gerth, T. Unruh, A. Rempel, H. Amenitsch and A. Magerl, Nucleation and growth of CdS nanoparticles observed by ultrafast SAXS, in Symposium VV - Advanced Materials Exploration with Neutrons and Synchrotron X-Rays, MRS Proceedings, 2013, vol. 1528, DOI: $10.1557 /$ opl.2013.572, http://journals.cambridge.org/ article_S1946427413005721.

15 B. Marmiroli, et al., Free jet micromixer to study fast chemical reactions by small angle X-ray scattering, $L a b$ Chip, 2009, 9, 2063-2069.

16 B. Marmiroli, et al., Experimental set-up for time resolved small angle X-ray scattering studies of nanoparticles formation using a free-jet micromixer, Nucl. Instrum. Methods Phys. Res., Sect. B, 2010, 268, 329-333.

17 H. Haberkorn, D. Franke, T. Frechen, W. Goesele and J. Rieger, Early stages of particle formation in precipitation reactions-quinacridone and boehmite as generic examples, J. Colloid Interface Sci., 2003, 259, 112-126.

18 A. Sirovich, J. E. M. L. Holmes, H. P. Keller, J. K. J. Mielke and C. S. P. K. R. Sreenivasan, Prandtl-Essentials of Fluid Mechanics, Springer, New York, 2010, vol. 158.

19 J. Nika Ilavsky, Software for two-dimensional data reduction, J. Appl. Crystallogr., 2012, 45, 324-328.

20 J. Ilavsky and P. R. Irena Jemian, Tool suite for modeling and analysis of small-angle scattering, J. Appl. Crystallogr., 2009, 42, 347-353.

21 J. P. Perdew, K. Burke and M. Ernzerhof, Generalized Gradient Approximation Made Simple, Phys. Rev. Lett., 1996, 77, 3865-3868.

22 J. P. Perdew, K. Burke and M. Ernzerhof, Generalized Gradient Approximation Made Simple [Phys. Rev. Lett., 77, 3865, 1996], Phys. Rev. Lett., 1997, 78, 1396-1396.

23 G. Kresse, Ab initio molecular dynamics for liquid metals, J. Non-Cryst. Solids, 1995, 192-193, 222-229.

24 G. Kresse and J. Hafner, Ab initio molecular-dynamics simulation of the liquid-metal-amorphous-semiconductor 
transition in germanium, Phys. Rev. B: Condens. Matter, 1994, 49, 14251-14269.

25 G. Kresse and J. Furthmüller, Efficiency of $a b$ initio total energy calculations for metals and semiconductors using a plane-wave basis set, Comput. Mater. Sci., 1996, 6, 15-50.

26 G. Kresse, Efficient iterative schemes for ab initio totalenergy calculations using a plane-wave basis set, Phys. Rev. B: Condens. Matter, 1996, 54, 11169-11186.

27 P. E. Blöchl, Projector augmented-wave method, Phys. Rev. B: Condens. Matter, 1994, 50, 17953-17979.

28 G. Kresse, From ultrasoft pseudopotentials to the projector augmented-wave method, Phys. Rev. B: Condens. Matter, 1999, 59, 1758-1775.

29 T. Proffen and R. B. Neder, DISCUS: a program for diffuse scattering and defect-structure simulation, J. Appl. Crystallogr., 1997, 30, 171-175.
30 A. J. C. Wilson, Seherrer after Sixty Years: A Survey and Some New Results in the Determination of Crystallite Size, 1978, pp. 102-113.

31 M. T. Landahl and E. Mollo-Christensen, Turbulence and random processes in fluid mechanics.

32 D. Gebauer, A. Völkel and H. Cölfen, Stable prenucleation calcium carbonate clusters, Science, 2008, 322, 1819-1822.

33 L. Ratke and P. W. Voorhees, Growth and Coarsening, Springer Berlin Heidelberg, 2002.

34 D. R. Lide and H. V. Kehiaian, CRC Handbook of Thermophysical and Thermochemical Data, 2012.

35 D. Gebauer, M. Kellermeier, J. D. Gale, L. Bergström and H. Cölfen, Pre-nucleation clusters as solute precursors in crystallisation, Chem. Soc. Rev., 2014, 43, 2348-2371.

36 T. Narayanan, High brilliance small-angle X-ray scattering applied to soft matter, Curr. Opin. Colloid Interface Sci., 2009, 14, 409-415. 